\title{
q-BERNOULLI AND EULERIAN NUMBERS
}

\author{
BY \\ L. CARLITZ
}

1. Introduction. In a previous paper [2] the writer defined a set of rational functions $\eta_{m}$ of the indeterminate $q$ by means of

$$
(q \eta+1)^{m}=\eta^{m} \cdot(m>1), \quad \eta_{0}=1, \quad \eta_{1}=0,
$$

and a set of polynomials

$$
\eta_{m}(x)=\eta_{m}(x, q)
$$

in $q^{x}$ by

$$
\eta_{m}(x)=\left([x]+q^{x} \eta\right)^{m}, \quad \eta_{m}(0)=\eta_{m},
$$

where $[x]=\left(q^{x}-1\right) /(q-1)$; also

$$
q^{x} \beta_{m}(x)=\eta_{m}(x)+(q-1) \eta_{m+1}(x), \quad \beta_{m}(0)=\beta_{m} .
$$

For $q=1, \beta_{m}$ reduces to the Bernoulli number $B_{m}, \beta_{m}(x)$ reduces to the Bernoulli polynomial $B_{m}(x) ; \eta_{m}$ however does not remain finite for $m>1$.

In the present paper we first define polynomials $A_{m s}=A_{m s}(q)$ by means of

$$
[x]^{m}=\sum_{s=1}^{m} A_{m s}\left[\begin{array}{c}
x+s-1 \\
m
\end{array}\right]
$$

where

$$
\left[\begin{array}{l}
x \\
m
\end{array}\right]=\frac{\left(q^{x}-1\right)\left(q^{x-1}-1\right) \cdots\left(q^{x-m+1}-1\right)}{(q-1)\left(q^{2}-1\right) \cdots\left(q^{m}-1\right)} .
$$

Alternatively if we define the rational function $H_{m}=H_{m}(x, q)$ by means of $H_{0}=1, H_{1}=1 /(x-q)$,

$$
(q H+1)^{m}=x H^{m}
$$

then we have

$$
\dot{H}_{m}(x, q)=A_{m}(x, q) / \prod_{s=1}^{m}\left(x-q^{s}\right)
$$

where

$$
A_{m}(x, q)=\sum_{e=1}^{m} A_{m s} x^{o-1}
$$

Presented to the Society, April 25, 1953; received by the editors February 5, 1953. 
and the coefficients are the same as those occurring in (1.4). For $q=1, A_{m s}$ and $H_{m}(x)$ reduce to well known functions; some of the properties of these quantities are stated in $\S 2$ below. As Frobenius [3] showed, many of the properties of the Bernoulli and related numbers can be derived from properties of $H_{m}$. We shall show that much the same is true in the case of the $q$ analogues.

In [2] a theorem somewhat analogous to the Staudt-Clausen theorem was obtained for $\beta_{m}$ (with $q$ an indeterminate). We now show that if $p$ is an odd prime and we put $q=a$, where the rational number $a$ is integral $(\bmod p)$, then if $a \equiv 1(\bmod p)$,

$$
p \beta_{m} \equiv-1(\bmod p)
$$

provided $p-1 \mid m$; otherwise $\beta_{m}$ is integral $(\bmod p)$. If $a \neq 1(\bmod p)$ the situation is more complicated. In particular, if $a$ is a primitive root $\left(\bmod p^{2}\right)$, then $\beta_{m}$ is integral $(\bmod p)$ for $p-1 \nmid m$, while for $p-1 \mid m$ we have

$$
p \beta_{m} \equiv-\frac{1}{k}(\bmod p), \quad\left(k=\left(a^{p-1}-1\right) / p\right) .
$$

In general the denominator of $\beta_{m}$ may be divisible by arbitrarily high powers of $p$ (see Theorem 4 below).

Finally we derive some congruences of Kummer's type for $H_{m}$, etc. For example if $q=a$ is integral $(\bmod p)$ while $x$ is an indeterminate, then

$$
H^{m}\left(H^{w}-1\right)^{r} \equiv 0\left(\bmod p^{m}, p^{r e}\right) \quad\left(p^{e-1}(p-1) \mid w\right),
$$

where after expansion of the left member $H^{k}$ is replaced by $H_{k}$. We also obtain simple congruences for the numbers $A_{m s}$ defined in (1.4). The corresponding results for $\eta_{m}$ and $\beta_{m}$ are more complicated.

2. Eulerian numbers. To facilitate comparison we quote the following formulas from the papers of Frobenius [3] and Worpitzky [5].

$$
\begin{aligned}
x^{m} & =\sum_{s=1}^{m} A_{m s}\left(\begin{array}{c}
x+s-1 \\
m
\end{array}\right) \quad(m \geqq 1), \\
A_{m+1, s} & =(m+2-s) A_{m, s-1}+s A_{m s}, \\
A_{m s} & =\sum_{r=0}^{s}(-1)^{r}\left(\begin{array}{c}
m+1 \\
r
\end{array}\right)(s-r)^{m}, \\
B_{m} & =\frac{1}{m+1} \sum_{r=1}^{m}(-1)^{m-r-1}\left(\begin{array}{c}
m \\
r-1
\end{array}\right)^{-1} A_{m r .}
\end{aligned}
$$

In the next place if we put

$$
A_{m}=A_{m}(x)=\sum_{e=1}^{m} A_{m s} x^{s-1},
$$


and let

$$
H_{m}=H_{m}(x)=(x-1)^{-m} R_{m}(x)
$$

then $H_{m}$ satisfies

$$
(H+1)^{m}=x H^{m}(m \geqq 1), \quad H_{0}=1 .
$$

The connection between $H_{m}$ and the Bernoulli numbers is furnished by

$$
\sum_{r=0}^{k-1} \zeta^{r} B_{m}\left(\frac{r}{k}\right)=\frac{k^{1-m} \zeta}{1-\zeta} m H_{m-1}(\zeta)
$$

where $\zeta^{k}=1, \zeta \neq 1$. An immediate consequence of $(2.7)$ is

$$
k^{m} B_{m}\left(\frac{r}{k}\right)-B_{m}=-m \sum_{\zeta \neq 1} \frac{1}{\zeta-1} H_{m-1}(\zeta),
$$

where $\zeta$ runs through the $k$ th roots of unity distinct from 1 .

We also mention

$$
H_{m}=\sum_{r=0}^{m}(x-1)^{-r} \Delta^{r} 0^{m}
$$

3. Some preliminaries. We shall use the notation of [2]; see in particular $\$ 2$ of that paper. In addition the following remarks will be useful. Let $f(u)$ be a polynomial in $q^{u}$ of degree $\leqq m$. Then the difference equation

$$
g(u+1)-c g(u)=f(u)
$$

has a unique polynomial solution $g(u)$, as can easily be proved by comparison of coefficients. To put the solution in more useful form we rewrite (3.1) as $(E-c) g(u)=f(u)$ and recall that

$$
\Delta=E-1, \Delta^{2}=(E-1)(E-q), \Delta^{3}=(E-1)(E-q)\left(E-q^{2}\right), \cdots .
$$

In the identity

$$
\begin{aligned}
\frac{1}{t-z}=\frac{1}{t-z_{1}} & +\frac{z-z_{1}}{t-z_{1}} \frac{1}{t-z_{2}}+\frac{\left(z-z_{1}\right)\left(z-z_{2}\right)}{\left(t-z_{1}\right)\left(t-z_{2}\right)} \frac{1}{t-z_{3}}+\cdots \\
& +\frac{\left(z-z_{1}\right) \cdots\left(z-z_{n}\right)}{\left(t-z_{1}\right) \cdots\left(t-z_{n}\right)} \frac{1}{t-z}
\end{aligned}
$$

take $t=c, z=E, z_{s}=q^{s-1}$, so that we get

$$
\begin{aligned}
\frac{1}{c-E}=\frac{1}{c-1} & +\frac{\Delta}{(c-1)(c-q)}+\frac{\Delta^{2}}{(c-1)(c-q)\left(c-q^{2}\right)}+\cdots \\
& +\frac{\Delta^{n}}{(c-1) \cdots\left(c-q^{n-1}\right)} \frac{1}{c-E} .
\end{aligned}
$$


Hence if we take $n>m$, we obtain the following formula for $g(u)$ :

$$
g(u)=\sum_{s=0}^{m} \frac{\Delta^{s} f(u)}{(c-1)(c-q) \cdots\left(c-q^{s}\right)} .
$$

4. The number $A_{m s}$. We suppose $A_{m s}$ defined by means of (1.4). Using the identity

$\left(q^{m+1}-1\right)\left(q^{x}-1\right)=\left(q^{m+1-s}-1\right)\left(q^{x+s}-1\right)+q^{m+1-s}\left(q^{s}-1\right)\left(q^{x+s-m-1}-1\right)$

and multiplying both members of (1.4) by $[x]$, we get

$$
\begin{aligned}
{[x]^{m+1} } & =\sum_{s} A_{m s}\left\{[m+1-s]\left[\begin{array}{c}
x+s \\
m+1
\end{array}\right]+q^{m+1-s}[s]\left[\begin{array}{c}
x+s-1 \\
m+1
\end{array}\right]\right\} \\
& =\sum_{s}\left[\begin{array}{c}
x+s-1 \\
m+1
\end{array}\right]\left\{[m+2-s] A_{m, s-1}+q^{m+1-s}[s] A_{m s}\right\},
\end{aligned}
$$

which implies the recursion

$$
A_{m+1, s}=[m+2-s] A_{m, s-1}+q^{m+1-s}[s] A_{m s} .
$$

For $q=1$ it is evident that (4.1) reduces to (2.2). As an immediate consequence of (4.1) we infer that $A_{m s}$ is a polynomial in $q$ with positive integral coefficients.

It is easy to show that $A_{m s}$ is divisible by $q^{(m-s)(m-s+1) / 2}$. Indeed if we put

$$
A_{m s}=q^{(m-s)(m-s+1) / 2} A_{m s}^{*}
$$

then (4.1) becomes

$$
A_{m+1,8}^{*}=[m+2-s] A_{m, 8-1}^{*}+[s] A_{m s}^{*},
$$

which proves the stated property. Moreover it follows easily from (4.3) that

$$
\operatorname{deg} A_{m s}^{*}=(s-1)(m-s) .
$$

Indeed assuming the truth of (4.4), we get

$$
\begin{aligned}
\operatorname{deg}\left([m+2-s] A_{m, s-1}^{*}\right) & =(m+1-s)+(s-2)(m+1-s) \\
& =(s-1)(m+1-s), \\
\ddots \operatorname{deg}\left([s] A_{m s}^{*}\right) & =(s-1)+(s-1)(m-s)=(s-1)(m+1-s),
\end{aligned}
$$

so that

$$
\operatorname{deg} A_{m+1, s}^{*}=(s-1)(m+1-s),
$$

which proves (4.4). 
The symmetry properties

$$
A_{m, m-s+1}^{*}=A_{m s}^{*}
$$

and

$$
A_{m s}^{*}(q)=q^{(s-1)(m-s)} A_{m s}^{*}\left(q^{-1}\right)
$$

will be proved below.

Comparing coefficients of $q^{m x}$ on both sides of (1.4) we get

$$
\sum_{s=1}^{m} A_{m s}=[m] !=[m][m-1] \cdots[1] .
$$

More generally if we expand both sides in powers of $q^{x}$ and equate coefficients we get

$$
\left[\begin{array}{c}
m \\
s
\end{array}\right] \sum_{r=1}^{m} A_{m r} q^{r s}=\left(\begin{array}{c}
m \\
s
\end{array}\right) q^{m s-s(s-1) / 2}[m] ! \quad(0 \leqq s \leqq m) .
$$

The following table of $A_{m s}^{*}, 1 \leqq s \leqq m \leqq 5$, is easily computed by means of (4.3).

\begin{tabular}{c||c|c|c|c|c}
\hline \hline & 1 & 2 & 3 & 4 & 5 \\
\hline 1 & 1 & & & & \\
2 & 1 & 1 & & & \\
3 & 1 & $2(q+1)$ & 1 & 1 & \\
4 & 1 & $3 q^{2}+5 q+3$ & $3 q^{2}+5 q+3$ & & \\
5 & 1 & $4 q^{3}+9 q^{2}+9 q+4$ & $6 q^{4}+16 q^{3}+22 q^{2}+16 q+6$ & $4 q^{3}+9 q^{2}+9 q+4$ & 1 \\
\hline
\end{tabular}

5. A formula for $A_{m r}^{*}$. It is easy to show that if $f(x)$ is a polynomial in $q^{x}$ of degree $\leqq m$,

$$
f(x)=\sum_{s=0}^{m} C_{m s}\left[\begin{array}{c}
x+s-1 \\
m
\end{array}\right]
$$

then

$$
\begin{gathered}
C_{m 0}=(-1)^{m} q^{m(m+1) / 2} f(0) \\
C_{m, m-r}=\sum_{s=0}^{r}(-1)^{s}\left[\begin{array}{c}
m+1 \\
s
\end{array}\right] f(r+1-s) q^{s(s-1) / 2}
\end{gathered}
$$

Since

$$
\sum_{s=0}^{m+1}(-1)^{s} q^{s(s-1) / 2}\left[\begin{array}{c}
m+1 \\
s
\end{array}\right] f(x+m+1-s)=0,
$$

we have in particular 


$$
\sum_{s=0}^{m+1}(-1)^{s} q^{s(s-1) / 2}\left[\begin{array}{c}
m+1 \\
s
\end{array}\right] f(r+1-s)=0,
$$

and (5.3) yields

$$
C_{m r}=\sum_{s=0}^{r}(-1)^{m-s} q^{(m-s)(m+1-s) / 2}\left[\begin{array}{c}
m+1 \\
s
\end{array}\right] f(s-r),
$$

which includes (5.2) also. Thus the coefficients in (5.1) are determined.

If we take $f(x)=[x]^{m}$, then $C_{m r}=A_{m r}$ and we get after a little manipulation

$$
A_{m r}^{*}=q^{r(r-1) / 2} \sum_{s=0}^{r}(-1)^{s} q^{s(s-1) / 2}\left[\begin{array}{c}
m+1 \\
s
\end{array}\right][r-s]^{m} ;
$$

for $q=1,(5.5)$ reduces to (2.3).

Replacing $q$ by $q^{-1}$, (5.5) becomes

$$
q^{(r-1)(m-r)} A_{m r}^{*}\left(q^{-1}\right)=q^{(r-1) m+r(r-1) / 2} \sum_{s=0}^{r}(-1)^{s} q^{s(s-1) / 2+e}\left[\begin{array}{c}
m+1 \\
s
\end{array}\right][r-s]^{m},
$$

where

$$
\begin{aligned}
e= & -\frac{s(s-1)-(m+1)(m+2)}{2}+\frac{(m+1-s)(m+2-s)}{2} \\
& +\frac{s(s+1)}{2}-(r-s+1) m=-(r-1) m .
\end{aligned}
$$

Hence

$$
A_{m r}^{*}(q)=q^{(r-1)(m-r)} A_{m r}^{*}\left(q^{-1}\right),
$$

which is identical with (4.6).

In the next place we observe that exactly as in the proof of (6.2) of [2] we have

$$
\begin{gathered}
\sum_{i=0}^{m}\left(\begin{array}{c}
m \\
i
\end{array}\right)[x]^{i+1} q^{(m-i) x} \frac{\eta_{m-i}}{i+1}+\left(q^{(m+1) x}-1\right) \frac{\eta_{m+1}}{m+1} \\
=\sum_{s=1}^{m} A_{m s} q^{m-s+1}\left[\begin{array}{c}
x+s-1 \\
m+1
\end{array}\right] .
\end{gathered}
$$

Divide both sides of this identity by $[x]$ and then put $x=0$. We find that

$$
\beta_{m}=\frac{1}{[m+1]} \sum_{s=1}^{m}(-1)^{m-s-1} q^{-(m-s)(m-s+1) / 2}\left[\begin{array}{c}
m \\
s-1
\end{array}\right]^{-1} A_{m s} .
$$

Using (4.2) and (4.5) this becomes 


$$
\begin{aligned}
\beta_{m} & =\frac{1}{[m+1]} \sum_{s=1}^{m}(-1)^{m-s-1}\left[\begin{array}{c}
m \\
s-1
\end{array}\right]^{-1} A_{m s}^{*} \\
& =\frac{1}{[m+1]} \sum_{s=1}^{m}(-1)^{s}\left[\begin{array}{c}
m \\
s
\end{array}\right]^{-1} A_{m s}^{*},
\end{aligned}
$$

the first of which may be compared with (2.4).

6. The polynomial $A_{m}(x)$. The polynomial $A_{m}(x)=A_{m}(x, q)$ is defined in (1.7) for $m \geqq 1$; we put $A_{0}(x)=1$. Put

$$
\phi_{m}(x)=\prod_{s=0}^{m}\left(x-q^{s}\right)
$$

and apply the Lagrange interpolation formula at the points $x=q^{s}$, $s=0,1, \cdots, m$. Since

$$
\begin{aligned}
\phi^{\prime}\left(q^{s}\right) & =\prod_{i=0}^{s-1}\left(q^{s}-q^{i}\right) \prod_{j=s+1}^{m}\left(q^{s}-q^{j}\right) \\
& =(-1)^{m-s} q^{m s-s(s-1) / 2}(q-1)^{m}[s] ![m-s] !
\end{aligned}
$$

we get using (4.8)

$$
A_{m}(x)=\frac{\phi_{m}(x)}{(q-1)^{m}} \sum_{s=0}^{m}(-1)^{m-s}\left(\begin{array}{c}
m \\
s
\end{array}\right) \frac{1}{x-q^{s}} .
$$

As a first application of (6.2) consider

$$
A_{m}\left(x^{-1}, q^{-1}\right)=\frac{x^{-m-1} q^{-m(m+1) / 2} \phi_{m}(x)}{q^{-m}(q-1)^{m}} \sum_{s=0}^{m}(-1)^{m-s}\left(\begin{array}{c}
m \\
s
\end{array}\right) \frac{x q^{s}}{x-q^{s}},
$$

which gives

$$
x^{m-1} q^{m(m-1) / 2} A_{m}\left(x^{-1}, q^{-1}\right)=A_{m}(x, q) \quad(m \geqq 1) .
$$

Substituting from (1.7) in (6.3) we get

$$
q^{m(m-1) / 2} \sum_{s=1}^{m} A_{m s}\left(q^{-1}\right) x^{m-s}=\sum_{s=1}^{m} A_{m s}(q) x^{s-1},
$$

which implies

$$
q^{m(m-1) / 2} A_{m s}\left(q^{-1}\right)=A_{m, m-s+1}(q) .
$$

Hence by (4.2) and (4.6), (6.4) becomes

$$
q^{m(m-1) / 2-(m-s)(m-s+1) / 2-(s-1)(m-s)} A_{m s}^{*}\left(q^{-1}\right)=q^{s(s-1) / 2} A_{m, m-s+1}^{*}(q),
$$

which is the same as (4.5).

7. The functions $H_{m}(x)$ and $H_{m}(u, x)$. Using (6.2) and (1.6) we get 


$$
(q-1)^{m} H_{m}(x)=(x-1) \sum_{s=0}^{m}(-1)^{m-s}\left(\begin{array}{c}
m \\
s
\end{array}\right) \frac{1}{x-q^{s}} .
$$

We remark that (7.1) implies

$$
H_{m}(x)=(x-1) \sum_{r=0}^{\infty} x^{-r-1}[r]^{m}
$$

for $|x|>\left|q^{s}\right|, 0 \leqq s \leqq m$. It is also evident that

$$
\begin{aligned}
(1+q H)^{m} & =(x-1) \sum_{r=0}^{m}\left(\begin{array}{c}
m \\
r
\end{array}\right) q^{r}(q-1)^{-r} \sum_{s=0}^{r}(-1)^{r-s}\left(\begin{array}{c}
r \\
s
\end{array}\right) \frac{1}{x-q^{s}} \\
& =(x-1) \sum_{s=0}^{m}\left(\begin{array}{c}
m \\
s
\end{array}\right) \frac{1}{x-q^{s}} \sum_{r=s}^{m}(-1)^{r-s}\left(\begin{array}{c}
m-s \\
r-s
\end{array}\right) q^{r}(q-1)^{-r} \\
& =(x-1) \sum_{s=0}^{m}\left(\begin{array}{c}
m \\
s
\end{array}\right) \frac{1}{x-q^{s}} \frac{q^{s}}{(q-1)^{s}}\left(\frac{-1}{q-1}\right)^{m-s} \\
& =(q-1)^{-m}(x-1) \sum_{s=0}^{m}(-1)^{m-s}\left(\begin{array}{c}
m \\
s
\end{array}\right) \frac{q^{s}}{x-q^{s}},
\end{aligned}
$$

which implies

$$
(1+q H)^{m}=x H^{m} \quad(m>1) .
$$

We have therefore proved (1.5). Alternatively taking (7.3) as definition of $H_{m}$ one can work back to the earlier formulas obtained for $A_{m s}$ above.

For some purposes it is convenient to define $H_{m}(u ; x)=H_{m}(u ; x, q)$, a polynomial in $q^{u}$. We put

$$
(q-1)^{m} H_{m}(u ; x)=(x-1) \sum_{s=0}^{m}(-1)^{m-s}\left(\begin{array}{c}
m \\
s
\end{array}\right) \frac{q^{s u}}{x-q^{s}},
$$

so that $H_{m}(0 ; x)=H_{m}(x)$. It follows at once from (7.4) that

$$
H_{m}\left(1-u ; x^{-1}, q^{-1}\right)=(-q)^{m} H_{m}(u ; x, q)
$$

and that

$$
x H_{m}(u ; x)-H_{m}(u+1 ; x)=(x-1)[u]^{m} .
$$

We have also

$$
\sum_{r=0}^{m}\left(\begin{array}{c}
m \\
r
\end{array}\right) q^{r} H_{r}(u ; x)=H_{m}(u+1 ; x),
$$

which becomes, using (7.6),

$$
(1+q H(u ; x))^{m}=x H_{m}(u ; x)-(x-1)[u]^{m} .
$$


For $u=0,(7.7)$ reduces to $(7.3)$.

Clearly (7.6) implies

$$
\sum_{i=0}^{k-1} x^{k-i}[u+i]^{m}=x^{k} H_{m}(u ; x)-H_{m}(u+k ; x),
$$

which includes (7.2) as a special case.

Since $H_{m}(u ; x)$ is a polynomial in $q^{u}$ of degree $m$, the remarks in $\$ 3$ apply to the difference equation (7.6). In particular, application of (3.2) leads to

$$
H_{m}(u ; x)=\sum_{s=0}^{m} \frac{\Delta^{s}[u]^{m}}{\psi_{s}(x)} \quad\left(\psi_{s}(x)=\prod_{r=1}^{s}\left(x-q^{r}\right)\right),
$$

provided $x \neq q^{r}, r=0,1, \cdots, m$. To simplify the right member of (7.9), we used (2.6) and (3.1) of [2]; thus

$$
\Delta^{s}[u]^{m}=\sum_{r=s}^{m} q^{r(r-1) / 2} a_{m, r}[r]_{s}[u]_{r-s} q^{s(u-r+s)}
$$

and (7.9) becomes after a little manipulation

$$
H_{m}(u ; x)=\sum_{r=0}^{m} q^{r(r-1) / 2} a_{m, r} \sum_{s=0}^{r} \frac{q^{s(u-r+s)}}{\psi_{s}(x)}[r]_{s}[u]_{r-s} .
$$

If we let $G_{r}(u)$ denote the inner sum it is clear from (3.2) that

$$
x G_{r}(u)-G_{r}(u+1)=[u]_{r} .
$$

In (7.10) put $u=0$, then

$$
H_{m}(x)=\sum_{r=0}^{m} q^{r(r-1) / 2} \frac{a_{m, r}[r] !}{\psi_{r}(x)},
$$

which for $q=1$ reduces to (2.9).

Using (7.1) and (7.4) it is easy to verify the formula

$$
H_{m}(u ; x)=\sum_{r=0}^{m}\left(\begin{array}{c}
m \\
r
\end{array}\right) q^{r u} H_{r}[u]^{m-r}=\left(q^{u} H+[u]\right)^{m} .
$$

Next using (7.12), (7.11), and the explicit formula [2, (6.2)] for $a_{m, 8}$ we get

$$
H_{m}(u ; x)=\sum_{r=0}^{m} \frac{1}{\psi_{r}(x)} \sum_{s=0}^{r}(-1)^{s} q^{s(s-1) / 2}\left[\begin{array}{l}
r \\
s
\end{array}\right][u+r-s]^{m},
$$

which is useful later.

8. Connection with $\eta_{m}(u)$. Using the formula $[2,(4.7)]$

$$
(q-1)^{m} \eta_{m}(u)=\sum_{s=0}^{m}(-1)^{m-s}\left(\begin{array}{c}
m \\
s
\end{array}\right) \frac{s}{[s]} q^{s u},
$$


we find that

$$
\begin{aligned}
\left(q^{k}-1\right)^{m-1} \sum_{r=0}^{k-1} \zeta^{r} \eta_{m}\left(u+\frac{r}{k}, q^{k}\right) & =\sum_{s=0}^{m}(-1)^{m-s}\left(\begin{array}{c}
m \\
s
\end{array}\right) \frac{s q^{k s u}}{\zeta^{-1} q^{s}-1} \\
& =m \sum_{s=0}^{m-1}(-1)^{m-1-s}\left(\begin{array}{c}
m-1 \\
s
\end{array}\right) \frac{\zeta q^{k s u+k u-1}}{q^{s}-\zeta q^{-1}}
\end{aligned}
$$

where

$$
\zeta^{k}=1, \quad \zeta \neq 1
$$

Comparing with (7.4) we have therefore

$$
[k]^{m-1} \sum_{r=0}^{k-1} \zeta^{r} \eta_{m}\left(u+\frac{r}{k}, q^{k}\right)=\frac{m \zeta q^{k u-1}}{1-\zeta} H_{m-1}\left(k u ; \zeta q^{-1}\right),
$$

and in particular for $u=0$

$$
[k]^{m-1} \sum_{r=0}^{k-1} \zeta^{r} \eta_{m}\left(\frac{r}{k}, q^{k}\right)=\frac{m \zeta q^{-1}}{1-\zeta} H_{m-1}\left(\zeta q^{-1}\right),
$$

which may be compared with (2.7).

Next using the multiplication formula (see $[2,(4.12)]$; note that a term is missing in that formula)

$$
[k]^{m-1} \sum_{r=0}^{k-1} \eta_{m}\left(u+\frac{r}{k}, q^{k}\right)=\eta_{m}(k u, q)+(-1)^{m} \frac{k-[k]}{(q-1)^{m}}
$$

together with (8.1) we get

$$
\begin{aligned}
k[k]^{m-1} \eta_{m}\left(u+\frac{r}{k}, q^{k}\right)-\eta_{m}(k u, q) & -(-1)^{m} \frac{k-[k]}{(q-1)^{m}} \\
& =\frac{m}{q} \sum_{\zeta \neq 1} \frac{\zeta^{r+1}}{1-\zeta} H_{m-1}\left(k u ; \zeta q^{-1}\right),
\end{aligned}
$$

and in particular for $u=0$,

$$
\begin{aligned}
k[k]^{m-1} \eta_{m}\left(\frac{r}{k}, q^{k}\right)-\eta_{m}-(-1)^{m} \frac{k-[k]}{(q-1)^{m}} & \quad \quad=\frac{m}{q} \sum_{\zeta \neq 1} \frac{\zeta^{+1}}{1-\zeta} H_{m-1}\left(\zeta q^{-1}\right) .
\end{aligned}
$$

By means of (1.3) it is easy to write down formulas like (8.1), $\cdots,(8.5)$ involving $\beta_{m}$.

9. Multiplication formulas. For the polynomial $H_{m}(u ; x)$ we have, using (7.4), 


$$
\begin{aligned}
\left(q^{k}-1\right)^{m} \sum_{r=0}^{k-1} \zeta^{r} q^{r t} & H_{m}\left(u+\frac{r}{k} ; \zeta q^{-k t}, q^{k}\right) \\
& =\left(\zeta q^{-k t}-1\right) \sum_{s=0}^{m}(-1)^{m-s}\left(\begin{array}{c}
m \\
s
\end{array}\right) \frac{q^{k s u}}{\zeta q^{-k t}-q^{k s}} \sum_{r=0}^{k-1} \zeta^{-r} q^{r(s+t)} \\
& =\left(\zeta-q^{k t}\right) \sum_{s=0}^{m}(-1)^{m-s}\left(\begin{array}{c}
m \\
s
\end{array}\right) \frac{q^{k s u}}{\zeta-q^{k(s+t)}} \frac{1-\zeta^{-k} q^{k(s+t)}}{1-\zeta^{-1} q^{s+t}}
\end{aligned}
$$

Consequently if $\zeta^{k-1}=1, \zeta \neq 1$, we get

$$
[k]^{m} \sum_{r=0}^{k-1} \zeta^{r} q^{r t} H_{m}\left(u+\frac{r}{k} ; \zeta q^{-k t}, q^{k}\right)=\frac{\zeta-q^{k t}}{\zeta-q^{t}} H_{m}\left(k u ; \zeta q^{-t}, q\right),
$$

analogous to (8.3).

In the special case $x=-q^{-1}$, the polynomial $\epsilon_{m}(u)$ of $[2, \S 8]$ satisfies

$$
\epsilon_{m}(u)=H_{m}\left(u ;-q^{-1}, q\right) ;
$$

in this case (9.1) becomes $(\zeta=-1, t=1)$

$$
[k]^{m} \sum_{r=0}^{k-1}(-q)^{r} \epsilon_{m}\left(u+\frac{r}{k}, q^{k}\right)=\frac{q^{k}+1}{q+1} \epsilon_{m}(k u, q)
$$

for $k$ odd; note that (8.6) of [2] requires a slight correction.

10. Staudt-Clausen theorems for $\beta_{m}$. In [2] a theorem analogous to the Staudt-Clausen theorem was proved for $\beta_{m}$ with $q$ indeterminate. Now on the other hand we replace $q$ by a rational number $a$ which is assumed to be integral modulo a fixed prime $p$. We shall use the representation $[2,(6.2)]$

$$
\beta_{m}=\sum_{s=0}^{m}(-1)^{s} a_{m, s}[s] ! /[s+1],
$$

where

$$
a_{m, s}=\frac{q^{-s(s-1) / 2}}{s !} \sum_{r=0}^{s}(-1)^{r} q^{r(r-1) / 2}\left[\begin{array}{l}
s \\
r
\end{array}\right][s-r]^{m}
$$

the quantity $a_{m, 8}$ is a polynomial in $q$ and has occurred in (7.10) and (7.11) above.

Suppose first that $a \equiv 1(\bmod p)$. Then from $(10.1)$ or $[2, \S 7]$ it is clear that the sth term in the right member of (10.1) is of the form $u_{s}$ $=N_{s}(a) / F_{s+1}(a)$, where $F_{s+1}(x)$ is the cyclotomic polynomial and $N_{s}(x)$ is a polynomial with integral coefficients. If we recall that $F_{k}(1)=p$ when $k=p^{e}$, $e \geqq 1$, but $F_{k}(1)=1$ otherwise, it is clear that $u_{s}$ is integral $(\bmod p)$ except possibly when $s+1=p^{e}$; the same holds also for $F_{k}(a)$. Now let $s+1=p^{e}$. Then by a simple computation it is seen that $[s]$ ! is divisible by exactly $p^{\prime}$, where 


$$
f=\left(p^{e}-1\right) /(p-1)-e,
$$

while the denominator is divisible by exactly $p^{e}$. Since $\left(p^{e}-1\right) /(p-1) \geqq 2 e$ for $e \geqq 2, p \geqq 3$, it follows that $u_{\mathrm{s}}$ is integral in this case. If $e=1, p \geqq 3$, we have first $p(a-1) /\left(a^{p}-1\right) \equiv 1(\bmod p)$. As for the numerator of $u_{p-1}$, it follows readily from (10.2) and

$$
\left[\begin{array}{c}
p-1 \\
r
\end{array}\right] \equiv\left(\begin{array}{c}
p-1 \\
r
\end{array}\right)(\bmod p)
$$

that

$$
\begin{aligned}
{[p-1] ! a_{m, p-1} } & \equiv \sum_{r=0}^{p-1}(-1)^{r}\left(\begin{array}{c}
p-1 \\
r
\end{array}\right) r^{m} \\
& \equiv \sum_{r=0}^{p-1} r^{m} \equiv\left\{\begin{aligned}
-1(\bmod p) & (p-1 \mid m), \\
0(\bmod p) & (p-1 \nmid m) .
\end{aligned}\right.
\end{aligned}
$$

We have therefore proved

TheOREM 1. Let $p \geqq 3, q=a \equiv 1(\bmod p)$. Then

$$
p \beta_{m} \equiv\left\{\begin{aligned}
-1(\bmod p) & (p-1 \mid m), \\
0(\bmod p) & (p-1 \nmid m) .
\end{aligned}\right.
$$

For $p=2$, the preceding argument shows that all terms in (10.1) are integral $(\bmod 2)$ except perhaps $u_{1}$ and $u_{3}$. Now

$$
u_{1}=\frac{a_{m, 1}}{[2]}=\frac{1}{a+1}
$$

while

$$
u_{3}=\frac{[3] ! a_{m, 3}}{[4]}=\frac{a^{-8}}{(a+1)\left(a^{2}+1\right)} \sum_{r=0}^{8}(-1)^{r} a^{r(r-1) / 2}\left[\begin{array}{c}
3 \\
r
\end{array}\right][3-r]^{m} .
$$

Let $2^{e} \mid(a+1), 2^{e+1} \nmid(a+1)$; then $\left(a^{2}+a+1\right)^{2} \equiv 1\left(\bmod 2^{\circ+1}\right)$ and

$$
\begin{aligned}
\sum_{r=0}^{3}(-1)^{r} a^{r(r-1) / 2}\left[\begin{array}{l}
3 \\
r
\end{array}\right][3-r]^{m} & \\
& \equiv\left(a^{2}+a+1\right)^{m}-\left(a^{2}+a+1\right)(a+1)^{m}+a\left(a^{2}+a+1\right) \\
& \equiv\left\{\begin{array}{cc}
0 & \left(\bmod 2^{e+1}\right) \\
a+1\left(\bmod 2^{e+1}\right) & (m \text { even}),
\end{array}\right.
\end{aligned}
$$

Consequently $u_{3}$ is integral $(\bmod 2)$ for $m$ even while for $m \operatorname{odd} 2 u_{3} \equiv 1(\bmod 2)$. This yields the following supplement to Theorem 1.

Theorem 2. Let $p=2, q=a \equiv 1(\bmod 2)$; also let $2^{a} \mid(a+1), 2^{\circ+1} \nmid(a+1)$. 
Then if $e=1$ we have $2 \beta_{m} \equiv 1(\bmod 2)$ for $m$ even $\geqq 2,2 \beta_{1} \equiv 1(\bmod 2)$, while $\beta_{m}$ is integral $(\bmod 2)$ for $m$ odd $\geqq 3$. If $e>1$ then

$$
2^{e} \beta_{m} \equiv 1(\bmod 2)
$$

for all $m \geqq 1$.

In particular it is evident from (10.4) that the denominator of $\beta_{m}$ may be divisible by arbitrarily high powers of 2 .

In the next place we suppose $q=a \neq 1(\bmod p), p>2$. It is now convenient to use $[2,(5.3)]$

$$
(q-1)^{m} \beta_{m}=\sum_{s=0}^{m}(-1)^{m-s}\left(\begin{array}{c}
m \\
s
\end{array}\right) \frac{s+1}{[s+1]} .
$$

We shall assume first that $a$ is a primitive root $\left(\bmod p^{2}\right)$. Clearly in the right member of (10.5) we need consider only those terms in which $p-1 \mid s+1$. Put $a^{p-1}=1+k p, p \nmid k$. Then

$$
\begin{gathered}
a^{(p-1) r}=1+r k p+\left(\begin{array}{c}
r \\
2
\end{array}\right) k^{2} p^{2}+\cdots \\
\frac{a^{(p-1) r}-1}{r p}=k+\frac{1}{2}(r-1) k^{2} p+\cdots \equiv k(\bmod p) .
\end{gathered}
$$

Thus (10.5) implies

$$
(a-1)^{m} p \beta_{m} \equiv(-1)^{m} \frac{1}{k} \sum_{r>0}\left(\begin{array}{c}
m \\
r(p-1)-1
\end{array}\right)(\bmod p) .
$$

But it is known $[4$, p. 255] that

$$
\sum_{0<r(p-1) \leqq m}\left(\begin{array}{c}
m \\
r(p-1)-1
\end{array}\right) \equiv\left\{\begin{aligned}
-1(\bmod p) & (p-1 \mid m), \\
0(\bmod p) & (p-1\} m) .
\end{aligned}\right.
$$

Hence (10.6) implies that $p$ is integral when $m \neq \equiv 0(\bmod p-1)$. This proves

THEOREM 3. Let $p \geqq 3, q=a, a$ primitive root $\left(\bmod p^{2}\right)$; then $\beta_{m}$ is integral $(\bmod p)$ for $p-1 \nmid m$, while

$$
p \beta_{m} \equiv-\frac{1}{k}(\bmod p) \quad(p-1 \mid m),
$$

where $k=\left(a^{p-1}-1\right) / p$.

It is now clear how to handle the general situation. We may state

Theorem 4. Let $p \geqq 3, q=a$, where $a$ belongs to the exponent $e(\bmod p)$, $e>1$. Put 


$$
a^{e}=1+p^{l} k
$$

Then

$$
(a-1)^{m} p^{l} \beta_{m} \equiv \frac{e}{k} \sum_{r>0}(-1)^{m-r e}\left(\begin{array}{c}
m \\
r e-1
\end{array}\right)(\bmod p) .
$$

In particular if $e=p-1$, then

$$
(a-1)^{m} p^{l} \beta \equiv\left\{\begin{aligned}
0(\bmod p) & (p-1 \nmid m), \\
-\frac{1}{k}(\bmod p) & (p-1 \mid m) .
\end{aligned}\right.
$$

To prove (10.9) it is only necessary to observe that (10.8) implies

$$
p^{l} \frac{r e}{a^{r e}-1}=p^{l} \frac{r e}{\left(1+p^{l} k\right)^{r}-1} \equiv \frac{e}{k}(\bmod p) .
$$

It is clear from (10.10) that the denominator of $\beta_{m}$ may be divisible by arbitrarily high powers of $p$. We also remark that theorems like Theorems 3 and 4 can be framed for $\eta_{m}$.

When $p^{e} \mid a$, it is evident from (10.5) that

$$
\beta_{m} \equiv \sum_{s=0}^{m}(-1)^{s}\left(\begin{array}{c}
m \\
s
\end{array}\right)(s+1) \equiv 0\left(\bmod p^{e}\right) \quad(m>1) .
$$

11. Congruences. The formula (7.11) together with (10.2) makes it possible to derive certain congruences satisfied by $H_{m}(x, q)$. We observe, to begin with, that if $q=a$ is integral $(\bmod p)$ then $u_{m}=[s] ! a_{m, s}$ satisfies, for $s$ fixed and $(p-1) p^{e-1} \mid w$,

$$
u^{m}\left(u^{w}-1\right)^{r} \equiv 0\left(\bmod p^{m}, p^{r e}\right),
$$

where after expansion of the left member, $u^{n}$ is replaced by $u_{n}$. To prove (11.1) we need only remark that

$$
u^{m}\left(u^{w}-1\right)^{r}=a^{-s(8-1) / 2} \sum_{r=0}^{s}(-1)^{r} a^{r(r-1) / 2}\left[\begin{array}{l}
s \\
r
\end{array}\right][s-r]^{m}\left([s-r]^{w}-1\right)^{r} .
$$

If we look on $x$ in (7.11) as an indeterminate and apply (11.1), we can assert that

$$
H^{m}\left(H^{w}-1\right)^{r} \equiv 0\left(\bmod p^{m}, p^{r e}\right) .
$$

We interpret this congruence in the following manner. The left member of (11.2) is a rational function of $x$ such that the coefficient of each term in the numerator $\equiv 0\left(\bmod p^{m}, p^{r}\right)$. We may call (11.2) Kummer's congruence for $H_{m}$. Using (7.13) we can prove like results for $H_{m}(u ; x)$, where $u$ is now an integer. 
In view of (1.6) the result (11.2) can be restated in terms of $A_{m}(x)$ :

$$
\sum_{s=0}^{r}(-1)^{r-s}\left(\begin{array}{l}
r \\
s
\end{array}\right) A_{m+s w}(x) \prod_{i=m+s w+1}^{m+r w}\left(x-a^{i}\right) \equiv 0\left(\bmod p^{m}, p^{r e}\right) .
$$

We may state

TheOREM 5. Let $q=a$ be integral $(\bmod p), x$ an indeterminate, and $r \geqq 1$; then (11.3) holds.

In the next place (11.3) implies congruences for the $A_{m, 8}$ of (1.7). (For the case $q=1$, compare [1].) Since

$$
\prod_{i=m+s w+1}^{m+r w}\left(x-a^{i}\right)=\prod_{i=0}^{(r-s) w}(-1)^{(r-s) w-i} a^{i(i+1) / 2+i(m+s w)}\left[\begin{array}{c}
(r-s) w \\
i
\end{array}\right] x^{i}
$$

examination of the coefficient of $x^{k-1}$ in (11.3) implies

$$
\text { 4) } \begin{aligned}
\sum_{s=0}^{r}(-1)^{r-s}\left(\begin{array}{l}
r \\
s
\end{array}\right) \sum_{i}(-1)^{(r-s) w-i} A_{m+s w, k-i} a^{i(i+1) / 2+i(m+s w)}\left[\begin{array}{c}
(r-s) w \\
i
\end{array}\right] \\
\equiv 0\left(\bmod p^{m}, p^{r e}\right) .
\end{aligned}
$$

In order to obtain simpler results we consider some special values of the parameters. In the first place we take $r=1$, so that (11.4) becomes

$$
A_{m+w, k}-\sum_{i}(-1)^{w-i} A_{m, k-i} a^{i(i+1) / 2+i m}\left[\begin{array}{c}
w \\
i
\end{array}\right] \equiv 0\left(\bmod p^{m}, p^{*}\right) .
$$

Now suppose first that $a \equiv 1(\bmod p)$. It is necessary to examine

$$
\left[\begin{array}{c}
w \\
i
\end{array}\right]=\frac{\left(a^{w}-1\right) \cdots\left(a^{w-i+1}-1\right)}{(a-1) \cdots\left(a^{i}-1\right)} .
$$

We assume from now on that $p>2$. If we put $a=1+p^{\imath} h, p \nmid h$, then as in the proof of Theorem 4 we find that

$$
\left[\begin{array}{c}
w \\
i
\end{array}\right] \text { and }\left(\begin{array}{c}
w \\
i
\end{array}\right)
$$

are divisible by exactly the same power of $p$. But if $i<p^{i}$, it is clear from the identity

$$
\left(\begin{array}{c}
w \\
i
\end{array}\right)=\frac{w}{i}\left(\begin{array}{c}
w-1 \\
i-1
\end{array}\right)
$$

that

$$
\left(\begin{array}{c}
w \\
i
\end{array}\right) \equiv 0\left(\bmod p^{-i}\right)
$$


Consequently if $p^{j-1} \leqq k<p^{j}$ and $j<e$, we see that (11.5) implies

$$
A_{m+w, k} \equiv A_{m k}\left(\bmod p^{m}, p^{e-j}\right) .
$$

This proves

Theorem 6. Let $p \geqq 3, q=a \equiv 1(\bmod p), p^{e-1}(p-1) \mid w$, and $p^{j-1} \leqq k<p^{j}$, where $j<e$. Then (11.7) holds.

When $a \neq 1(\bmod p)$ let $a$ belong to the exponent $t(\bmod p)$. Then it is clear from (11.6) that we need only consider those factors in the right member with exponents divisible by $t$. Thus if $i_{0}$ is the greatest integer $\leqq i / t$ we need only examine

$$
\left[\begin{array}{c}
w / t \\
i_{0}
\end{array}\right]
$$

with $a$ replaced by $a^{t}$. The preceding discussion therefore applies and we obtain the following theorem which includes Theorem 6 .

THEOREM 7. Let $p \geqq 3$, let $q=a$ belong to the exponent $t(\bmod p), p^{\circ-1}(p-1) \mid w$ and $k<t p^{i}$, where $j<e$. Then (11.7) holds.

The case $k=w$ is not covered by the theorem. We find for example that if $w=t=p-1($ so that $a$ is a primitive root $(\bmod p))$, then

$$
A_{m+p-1, k} \equiv\left\{\begin{array}{ccc}
A_{m, k} & (\bmod p) & (k<p-1), \\
A_{m, p-1}+\left(\frac{a}{p}\right) A_{m, 0} & (\bmod p) & (k=p-1),
\end{array}\right.
$$

where $(a / p)$ is Legendre's symbol.

Returning to (11.2) we can also consider the case in which $x$ is put equal to an integer $(\bmod p)$, provided the resulting denominators are not divisible by $p$. Now the least common denominator is evidently

$$
\psi_{m+r w}(x)=\prod_{s=1}^{m+r w}\left(x-a^{s}\right) .
$$

It will therefore suffice to assume that $x \not \equiv a^{s}(\bmod p)$ for any $s$. We may therefore state

THEOREM 8. Let $a$ and $x$ be rational numbers that are integral $(\bmod p)$ and suppose that $x \neq a^{s}(\bmod p)$ for any s. Let

$$
p^{-1}(p-1) \mid w \text { and } r \geqq 1 .
$$

Then

$$
H^{m}(x)\left(H^{m}(x)-1\right)^{r} \equiv 0\left(\bmod p^{m}, p^{r e}\right) .
$$


In particular the theorem may be applied with slight changes to $\epsilon_{m}(u)$ $=\epsilon_{m}(u, a)$ defined in $(9.2)$; we have explicitly [2, (8.18) ]

$$
\begin{aligned}
\epsilon_{m}(u)=\sum_{s=0}^{m} \frac{(-1)^{s} a^{s}}{(a+1)\left(a^{2}+1\right) \cdots\left(a^{s+1}+1\right)} \\
\cdot \sum_{r=0}^{s}(-1)^{r} a^{r(r-1) / 2}\left[\begin{array}{l}
s \\
r
\end{array}\right][u+s-r]^{m},
\end{aligned}
$$

which is included in (7.13). If $u$ is an integer we have

$$
\epsilon^{m}(u)\left(\epsilon^{w}(u)-1\right)^{r} \equiv 0\left(\bmod p^{m}, p^{r e}\right)
$$

provided $p \equiv 3(\bmod 4)$ and $a$ is a quadratic residue $(\bmod p)$. For in this case -1 is a nonresidue $(\bmod p)$ and therefore $-1 \not a^{s}$ for any $s$.

12. Congruences involving $\eta_{m}$ and $\beta_{m}$. Let

$$
\omega_{m}=\omega_{m, k, r}=\frac{1}{m}\left\{k[k]^{m-1} \eta_{m}\left(\frac{r}{k}, q^{k}\right)-\eta_{m}-(-1)^{m} \frac{k-[k]}{(q-1)^{m}}\right\}
$$

so that by (8.5)

$$
\omega_{m}=\frac{1}{q} \sum_{\zeta \neq 1} \frac{\zeta^{++1}}{1-\zeta} H_{m-1}\left(\zeta q^{-1}\right),
$$

where $\zeta$ runs through the $k$ th roots of unity distinct from 1 . As for the denominators in the right member of (12.2), note that

$$
\prod_{\zeta \neq 1}\left(a^{s}-\zeta\right)=\frac{a^{k s}-1}{a^{s}-1}=a^{s(k-1)}+\cdots+a^{s}+1,
$$

which is prime to $p$ for all $s$ provided $p \nmid k$. We may therefore state

THEOREM 9. If $a$ is integral $(\bmod p)$ and $p \nmid k$, then

$$
\omega^{m}\left(\omega^{w}-1\right)^{r} \equiv 0\left(\bmod p^{m-1}, p^{r e}\right),
$$

where $\omega_{m}$ is defined by (12.1) and $p^{e-1}(p-1) \mid w$.

As for $\beta_{m}$ we have

$$
\begin{aligned}
k[k]^{m-1} q^{r} \beta_{m}\left(\frac{r}{k}, q^{k}\right)-\beta_{m} & \\
= & \frac{(m+1)(q-1)}{q} \sum_{\zeta \neq 1} \frac{\zeta^{r+1}}{1-\zeta} H_{m}\left(\zeta q^{-1}\right)+\frac{m}{q} \sum_{\zeta \neq 1} \frac{\zeta^{r+1}}{1-\zeta} H_{m-1}\left(\zeta q^{-1}\right)
\end{aligned}
$$

analogous to (8.5). In much the same way as above (12.4) implies

Theorem 10. If a is integral $(\bmod p)$ and $p \nmid k$ then

$$
\Omega^{m}\left(\Omega^{\infty}-1\right)^{r} \equiv 0\left(\bmod p^{m-1}, p^{(r-1) e}\right),
$$


where $\Omega_{m}$ stands for the left member of (12.4) and $p^{\circ-1}(p-1) \mid w$.

Unfortunately we seem unable to obtain simpler congruences for $\beta_{m}$ and $\eta_{m}$.

13. Combinatorial interpretation of $a_{m s r}$. Put

$$
A_{m s}^{*}=\sum_{r=0}^{(s-1)(m-s)} a_{m s r_{0}} q^{r} \quad\left(r_{0}=s(s-1) / 2+r\right) .
$$

The following combinatorial interpretation of the coefficients $a_{m s r}$ was kindly suggested by J. Riordan. The number $a_{m e r}$ is the number of permutations of $m$ things requiring $s$ readings and such that $r=r_{2}+2 r_{3}+\cdots+(s-1) r_{s}$, where $r_{k}$ is the number of elements read on the $k$ th reading. The following numerical illustration for $m=4$ was also supplied by Riordan.

\begin{tabular}{|c|c|c|c|}
\hline Permutation & Reading & $s$ & $r$ \\
\hline 1234 & 1234 & 1 & 0 \\
\hline $\begin{array}{l}1243 \\
1423 \\
4123\end{array}$ & $123 \mid 4$ & 2 & 1 \\
\hline $\begin{array}{l}1324 \\
1342 \\
3124 \\
3142 \\
3412\end{array}$ & $12 \mid 34$ & 2 & 2 \\
\hline $\begin{array}{l}2134 \\
2314 \\
2341\end{array}$ & $1 \mid 234$ & 2 & 3 \\
\hline $\begin{array}{l}1432 \\
4132 \\
4312\end{array}$ & $12|3| 4$ & 3 & 3 \\
\hline $\begin{array}{l}2134 \\
2413 \\
2431 \\
4213 \\
4231\end{array}$ & $1|23| 4$ & 3 & 4 \\
\hline $\begin{array}{l}3214 \\
3241 \\
3421\end{array}$ & $1|2| 34$ & 3 & 5 \\
\hline 4321 & $1|2| 3 \mid 4$ & 4 & 6 \\
\hline
\end{tabular}




\section{REFERENCES}

1. L. Carlitz and J. Riordan, Congruences for the Eulerian numbers, Duke Math. J. vol. 20 (1953) pp. 339-344. 1000.

2. L. Carlitz, q-Bernoulli numbers and polynomials, Duke Math. J. vol. 15 (1948) pp. 987-

3. G. Frobenius, Über die Bernoullischen Zahlen und die Eulerschen Polynome, Preuss. Akad. Wiss. Sitzungsber. (1910) pp. 809-847.

4. N. Nielsen, Sur le theorème de v. Staudt et de Th. Clausen relatif aux nombres de Bernoulli, Annali di Matematica (3) vol. 22 (1914) pp. 249-261.

5. J. Worpitzky, Studient ïber die Bernoullischen und Eulerschen Zahlen, J. Reine Angew. Math. vol. 94 (1883) pp. 202-232.

DUKE UNIVERSITY,

DURHAM, N. C. 